\title{
Precision Measurements of Top Quark Production with the ATLAS Detector
}

\author{
Philipp Stolte ${ }^{1, a}$ on behalf of the ATLAS Collaboration \\ ${ }^{1}$ II. Physikalisches Institut, Georg-August-Universität Göttingen, Friedrich-Hund-Platz 1, D-37077 Göttingen, \\ Germany
}

\begin{abstract}
The top quark is the heaviest known fundamental particle. It is the only quark that decays before it hadronises which gives us the unique opportunity to probe the properties of bare quarks and to test perturbative QCD. This overview will focus on a few recent precision top quark measurements by the ATLAS Collaboration at the LHC: Fiducial top pair and single top production cross-sections including differential distributions will be presented and compared with QCD predictions. The results include the first top quark measurements at $13 \mathrm{TeV}$ using data from LHC Run 2.
\end{abstract}

\section{Introduction}

The top quark is known to be the heaviest particle in the Standard Model (SM) of elementary particle physics, more than 20 years after it has been discovered at the Tevatron in 1995 [1,2]. Due to its large mass, the lifetime of the top quark is extremely short which allows probing the properties of bare quarks and testing perturbative QCD. Several measurements of top quark cross-sections are discussed in the following. Those measurements do not only serve to perform precision tests of QCD to high orders, but as most models for new physics involve top quarks, its understanding is also important for future searches. The first part of this overview covers measurements of top quark pairs $(t \bar{t})$, the second focuses on single top quark measurements including both inclusive and differential measurements with the ATLAS detector [3] at the Large Hadron Collider (LHC) [4] at CERN.

Top quarks decay predominantly into a $W$ boson and a $b$ quark. As the $W$ boson can decay hadronically into two quarks or leptonically into a charged lepton $\ell$ and the corresponding neutrino $v_{\ell}$, three decay channels of $t \bar{t}$ pairs need to be distinguished. If both $W$ bosons decay hadronically, the decay channel is called all-hadronic and has the largest branching ratio (BR) but suffers from a large amount of multijet background. The dilepton channel comprises events where both $W$ bosons decay leptonically. This channel has a very good signal-over-background ratio but only a low BR. The lepton+jets channel with one $W$ boson decaying leptonically and one hadronically is often regarded as a compromise between a high BR and a reasonable signal-over-background ratio. In the next section, a $t \bar{t}$ inclusive cross-section measurement performed at $13 \mathrm{TeV}$ is presented, followed by differential crosssection measurements in the dilepton and the lepton+jets decay channel at centre-of-mass energies of 7, 8 and $13 \mathrm{TeV}$. The latter measurements serve as important tests of QCD, of various possible Monte Carlo (MC) generators and of parton distribution functions (PDFs).

\footnotetext{
a e-mail: pstolte@cern.ch
} 
Single top quarks are produced in three different ways. $t$-channel, $s$-channel and $W t$ production can be distinguished. The related leading order (LO) Feynman diagrams are illustrated in Fig. 1. While $t$-channel and $s$-channel processes have already been measured at the Tevatron [5-8], evidence for $W t$ production was first reported by ATLAS [9] and CMS [10] at $7 \mathrm{TeV}$ in 2012.

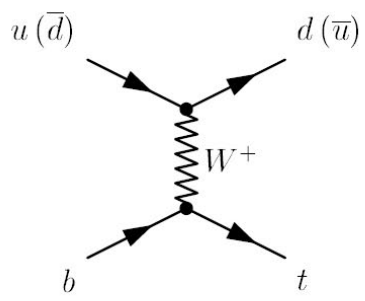

(a)

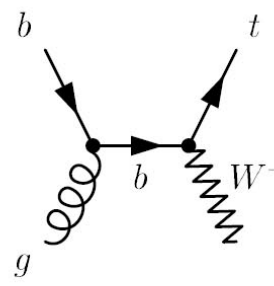

(b)

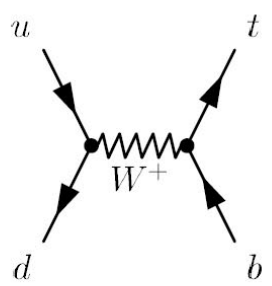

(c)

Figure 1. Single top production: (a) $t$-channel, (b) $W t$ and (c) $s$-channel production.

Single top inclusive cross-section measurements in proton-proton collisions at centre-of-mass energies of 8 and $13 \mathrm{TeV}$ are described in Sections 6-8.

\section{Top Quark Pair Inclusive Production Cross-Section at $13 \mathrm{TeV}$}

The latest inclusive cross-section measurement of $t \bar{t}$ pairs uses a data sample with an integrated luminosity of $\mathcal{L}=3.2 \mathrm{fb}^{-1}$ of proton-proton collisions at a centre-of-mass energy of $\sqrt{s}=13 \mathrm{TeV}$, collected in 2015 by the ATLAS detector [11]. The analysis is based on dileptonic $e \mu$ events, i.e. the selection requires $t \bar{t}$ events with exactly one electron, one muon and one or two $b$-tagged jets. The inclusive cross-section is calculated by counting the number of opposite-sign $e \mu$ events with exactly one $\left(N_{1}\right)$ and exactly two $\left(N_{2}\right) b$-tagged jets. These two event numbers can be expressed via the following formulae:

$$
\begin{aligned}
& N_{1}=\mathcal{L} \sigma_{t \bar{t}} \varepsilon_{e \mu} 2 \varepsilon_{b}\left(1-C_{b} \varepsilon_{b}\right)+N_{1}^{\mathrm{bkg}} \\
& N_{2}=\mathcal{L} \sigma_{t \bar{t}} \varepsilon_{e \mu} C_{b} \varepsilon_{b}^{2}+N_{2}^{\mathrm{bkg}} .
\end{aligned}
$$

The yields amount to $N_{1}=11958$ and $N_{2}=7069$ for the events in data and $N_{1}^{\text {bkg }}=1370 \pm 120$ and $N_{2}^{\mathrm{bkg}}=340 \pm 88$ for the number of background events. A preselection efficiency for leptons $\varepsilon_{e \mu}=0.83 \%$ and a correlation coefficient $C_{b}=\varepsilon_{b b} / \varepsilon_{b}^{2}=1.002$ enter the equations as well. Unknown are the cross-section $\sigma_{t \bar{t}}$ and the combined jet probability $\varepsilon_{b}$ which contains selection, reconstruction and $b$-tagging information. These two values can be obtained directly from solving the two equations. The combined probability for a jet from one of the top quarks to be within the detector acceptance and tagged as a $b$ jet is measured to be $\varepsilon_{b}=0.559 \pm 0.004$ (stat) \pm 0.003 (syst) which agrees with the theoretical calculation: $\varepsilon_{b}^{\mathrm{MC}}=0.549$. The resulting cross-section is $\sigma_{t \bar{t}}=818 \pm 8$ (stat) \pm 27 (syst) \pm 19 (lumi) \pm 12 (beam) pb, consistent with the theoretical prediction based on NNLO+NNLL (next-tonext-to-leading order + next-to-next-to-leading logarithmic order): $832_{-46}^{+40} \mathrm{pb}$. The total uncertainty from the measured cross-section is calculated to be $36 \mathrm{pb}$, which is smaller than the uncertainty from the theoretical prediction. The dominant systematic uncertainties arise from $t \bar{t}$ modelling, in particular shower and hadronisation uncertainties have a large impact. The cross-section result is visualised with other ATLAS measurements at lower centre-of-mass energies in Fig. 2. 


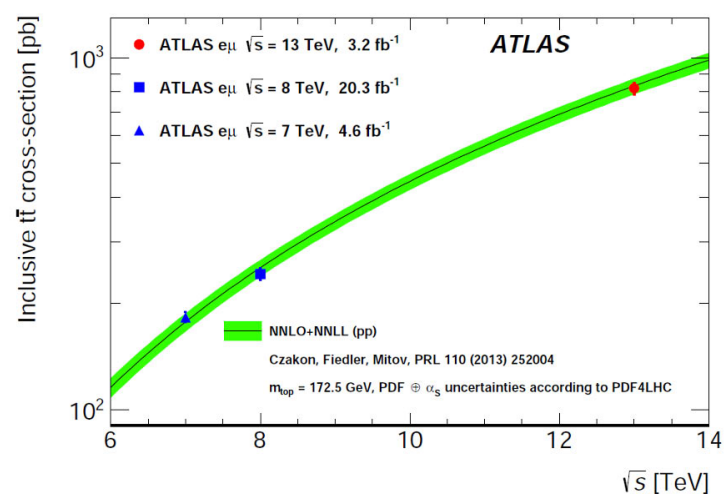

Figure 2. Inclusive $t \bar{t}$ cross-section measurements at different centre-of-mass energies using the ATLAS detector. The measured values are compared with a theoretical NNLO+NNLL calculation [11].

\section{Top Quark Pair Differential Cross-Sections in the Dilepton Channel at $7 \mathrm{TeV}$ and $8 \mathrm{TeV}$}

This differential measurement of top quark pair cross-sections has been performed in the dilepton channel using proton-proton collisions at centre-of-mass energies of $\sqrt{s}=7$ and $8 \mathrm{TeV}$ [12]. The dataset corresponds to integrated luminosities of $\mathcal{L}=4.6 \mathrm{fb}^{-1}$ at $7 \mathrm{TeV}$ and $\mathcal{L}=20.2 \mathrm{fb}^{-1}$ at $8 \mathrm{TeV}$. The analysis focuses on three different observables of $t \bar{t}$ pairs: the transverse momentum $p_{\mathrm{T}}^{t \bar{t}}$, the invariant mass $m^{t \bar{t}}$ and the rapidity $\left|y^{t \bar{t}}\right|$ of the $t \bar{t}$ system. Events are selected in the dilepton final state, requiring exactly two charged leptons and at least two jets with at least one $b$-tagged jet. The analysis using $7 \mathrm{TeV}$ data exploits all possible leptonic final states, namely the $e e$, $e \mu$ and $\mu \mu$ channel, while the $8 \mathrm{TeV}$ measurement is limited to $e \mu$ events to reduce the background contribution from $Z+$ jets decays. The four-momentum of the $t \bar{t}$ system is reconstructed from the required objects, i.e. two charged leptons, two jets and missing transverse energy due to the two neutrinos. In order to determine differential cross-sections from reconstructed events, an unfolding method is employed. The observables are unfolded to parton level using a Bayesian iterative unfolding method [13] implemented in the RooUnfold package [14] based on a migration matrix. The dominant systematic uncertainties originate from $t \bar{t}$ modelling, especially parton shower and generator uncertainties.

The resulting differential distributions for the three chosen observables are shown in Fig. 3. The predictions from MC simulation and QCD calculation agree with data in a wide range of kinematic distributions. In particular the measured distributions for $m^{t \bar{t}}$ are well described by the predictions for both $7 \mathrm{TeV}$ and $8 \mathrm{TeV}$. The $\left|y^{t \bar{t}}\right|$ distributions are sensitive to the choice of underlying PDF sets and show a certain tension between data and the full NNLO prediction at high rapidity values. The resulting distributions for $p_{\mathrm{T}}^{t \bar{t}}$ reveal a disagreement between data and NLO+NNLL (next-to-leading order + next-to-next-to-leading logarithmic order) predictions for both analysed centre-of-mass energies. Sensitivity to the choice of the generator, to scale settings and the level of radiation (initial and final state radiation) is measured as well: The predictions tend to have harder transverse momentum distributions than data. A comparison of different generator settings exhibits that $p_{\mathrm{T}}^{t \bar{t}}$ is well described by Powheg+Pythia with $h_{\text {damp }}=m_{t}$ and MC@NLO+Herwig but poorly described by Powheg+Pythia with $h_{\mathrm{damp}}=\infty$, where $h_{\mathrm{damp}}$ is a parameter that regulates the high- $p_{\mathrm{T}}$ radiation in Powheg. 

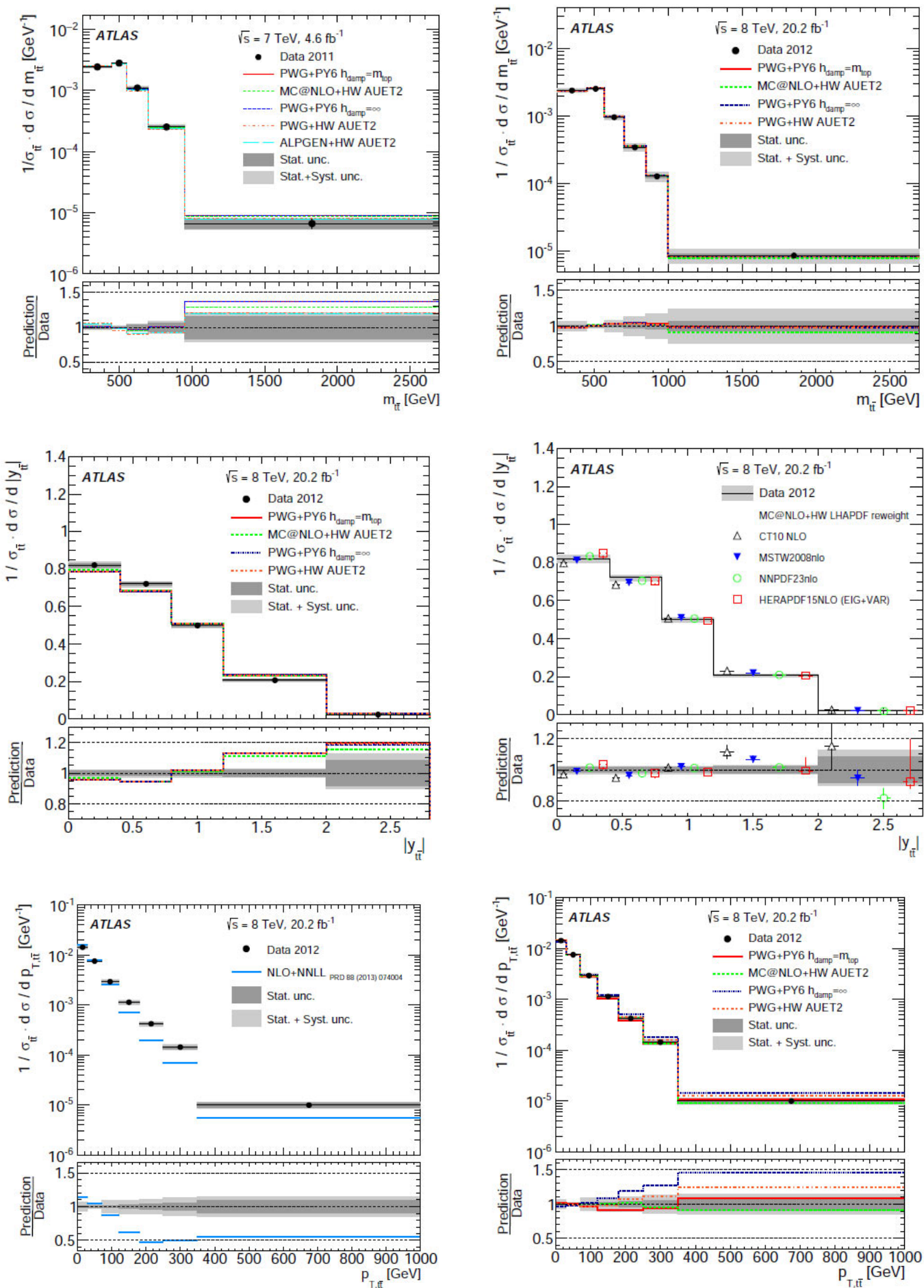

Figure 3. Differential $t \bar{t}$ cross-section measurements at $\sqrt{s}=7$ and $8 \mathrm{TeV}$ for three different $t \bar{t}$ observables: the transverse momentum $p_{\mathrm{T}}^{t \bar{t}}$, the invariant mass $m^{t \bar{t}}$ and the rapidity $\left|y^{t \bar{t}}\right|$ of the $t \bar{t}$ system. The bottom panel contains the ratio of prediction to data [12]. 


\section{Top Quark Pair Differential Cross-Sections in the Lepton+Jets Channel at $8 \mathrm{TeV}$}

Another measurement of differential cross-sections of $t \bar{t}$ production at a centre-of-mass energy of $8 \mathrm{TeV}$ has been performed in the lepton+jets decay channel [15]. The dataset corresponds to an integrated luminosity of $\mathcal{L}=20.3 \mathrm{fb}^{-1}$, recorded in 2012 in proton-proton collisions with the ATLAS detector. The measured cross-sections are presented as a function of observables of the $t \bar{t}$ system, the hadronically decaying top quark as well as event level kinematics. The analysis is performed in both the fiducial particle-level phase space and the full phase space. According to the requirements of the lepton+jets decay channel of $t \bar{t}$ pairs, events with exactly one lepton matched to the trigger object and at least four jets out of which two are $b$-tagged are selected. The chosen observables are unfolded to particle and parton level using a Bayesian iterative unfolding method as already introduced in Sec. 3 for the corresponding dilepton analysis. The migration matrices reveal a high level of diagonality; the correlations between particle and detector level reach around $85-95 \%$.

Results of this cross-section measurement for different MC generators are shown in Fig. 4. The discussion mainly focuses on the observables $p_{\mathrm{T}}^{t \text {,had }}$ and $\left|y^{t, \text { had }}\right|$ of the hadronically decaying top quark as well as on the transverse momentum $p_{\mathrm{T}}^{t \bar{t}}$, the invariant mass $m^{t \bar{t}}$ and the rapidity $\left|y^{t \bar{t}}\right|$ of the $t \bar{t}$ system, as they have been used in the related dilepton analysis. In general, the results are in fair agreement with the predictions over a wide kinematic range. Nevertheless, most generators predict harder $p_{\mathrm{T}}$ distributions at large values exceeding $400 \mathrm{GeV}$ compared to the observations made with data. The harder $p_{\mathrm{T}}$ spectrum in data cannot be fully explained by electroweak corrections which are missing in the given MC generator calculations. The rapidity-based observables show only a modest agreement between the predictions and data, while the agreement is acceptable for most generators in the $m^{t \bar{t}}$ and $p_{\mathrm{T}}^{t \bar{t}}$ distributions. The results underline that setting $h_{\mathrm{damp}}=m_{t}$ improves the agreement in all Powheg samples.

In Figure 5, the normalised $t \bar{t}$ full phase space differential cross-sections are compared with theoretical higher-order QCD calculations. Four calculations that offer beyond-NLO accuracy are considered in the analysis: an approximate next-to-next-to-leading order (aNNLO), an approximate next-tonext-to-next-to-leading order $\left(\mathrm{aN}^{3} \mathrm{LO}\right)$, an approximate NLO+NNLL and a full NNLO calculation. The studies reveal that the first two approximate calculations agree very poorly with the $p_{\mathrm{T}}^{t \text {,had }}$ spectrum while for other observables like $\left|y^{t \text {,had }}\right|$ an improvement of the $\mathrm{aN}^{3} \mathrm{LO}$ calculation with respect to aNNLO becomes obvious. NLO+NNLL precision leads to a better agreement for most observables whereas a large discrepancy between data and calculation can still be observed for $p_{\mathrm{T}}^{t \bar{t}}$. Full NNLO precision is required to reach a good agreement between data and QCD calculation indicating that the disagreement for simpler approximate calculations or generator predictions is due to missing higher-order terms.

\section{Top Quark Pair Differential Cross-Sections in the Lepton+Jets Channel at $13 \mathrm{TeV}$}

The measurement of $t \bar{t}$ differential cross-sections in the lepton+jets decay channel has been repeated at a centre-of-mass energy of $\sqrt{s}=13 \mathrm{TeV}$ [16]. The integrated luminosity corresponds to $\mathcal{L}=3.2 \mathrm{fb}^{-1}$, recorded in 2015 in proton-proton collisions with the ATLAS detector. The measurement concentrates on five kinematic observables as they have already been analysed in the corresponding $8 \mathrm{TeV}$ measurement: $p_{\mathrm{T}}^{t \text {,had }}$ and $\left|y^{t \text {,had }}\right|$ of the hadronically decaying top quark, the transverse momentum $p_{\mathrm{T}}^{t \bar{t}}$, the invariant mass $m^{t \bar{t}}$ and the rapidity $\left|y^{t \bar{t}}\right|$ of the $t \bar{t}$ system. Two separate selections are applied that each focus on different momentum phase spaces of the top quark, denoted as resolved and boosted 

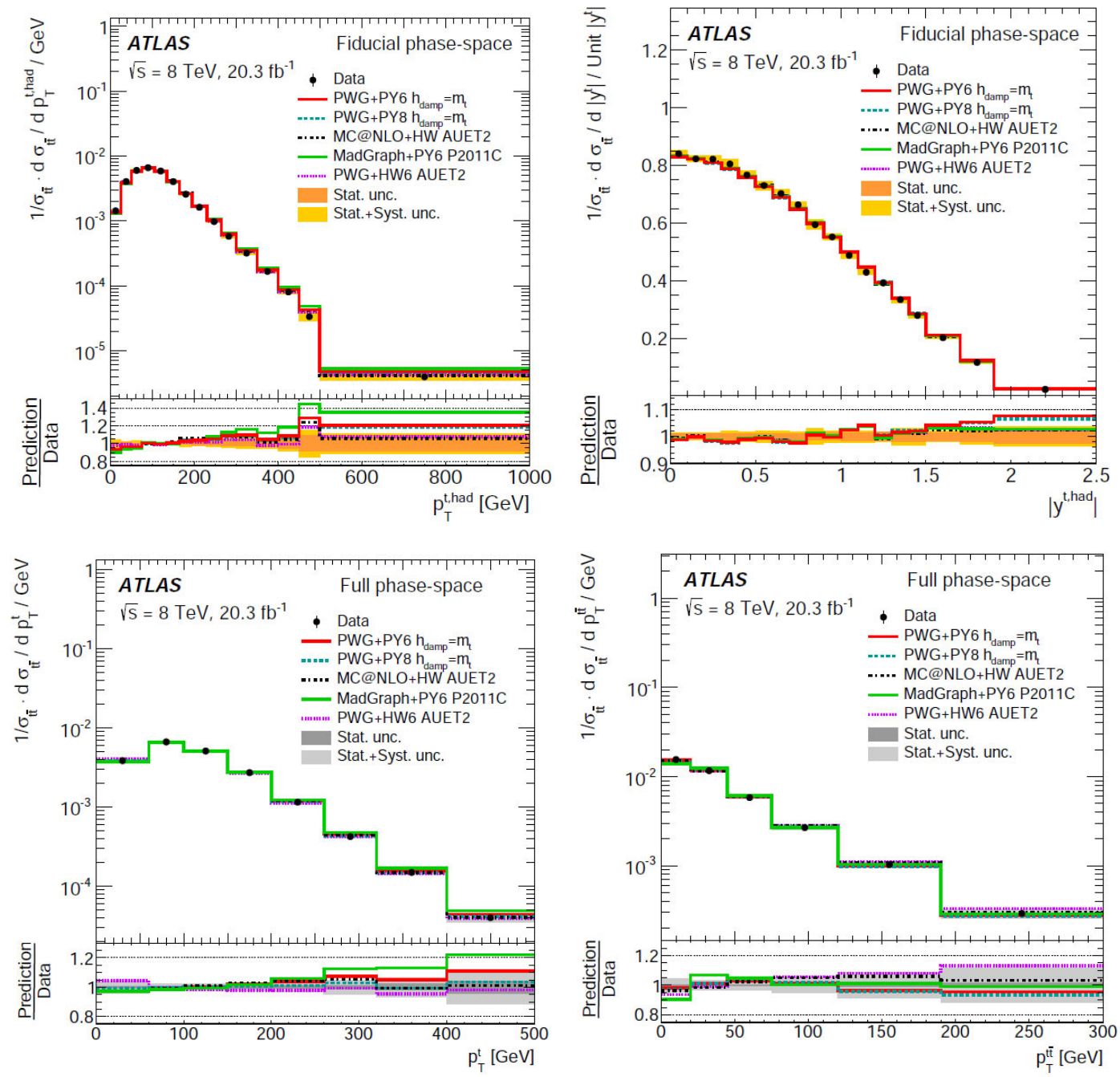

Figure 4. Differential $t \bar{t}$ cross-section measurements at $\sqrt{s}=8 \mathrm{TeV}$ compared with different MC generators. Distributions for three different observables are shown: $p_{\mathrm{T}}^{t \text {,had }}$ in the fiducial and full phase space as well as $\left|y^{\text {thad }}\right|$ in the fiducial and $p_{\mathrm{T}}^{t \bar{t}}$ in the full phase space. The bottom panel contains the ratio of prediction to data [15].

topology. Events in the resolved regime are characterised by one charged lepton, at least four jets and at least two jets out of these four which are $b$-tagged. The four-momenta of the leptonically and hadronically decaying top quarks and their complete decay chain are reconstructed from final state objects. Boosted top quark decay events are composed of one charged lepton, at least one small- $R$ jet and exactly one top-tagged large- $R$ jet. All these jets need to be $b$-tagged. The large- $R$ jet is directly identified as the hadronic top quark candidate. The measurement is performed on particle level; the 

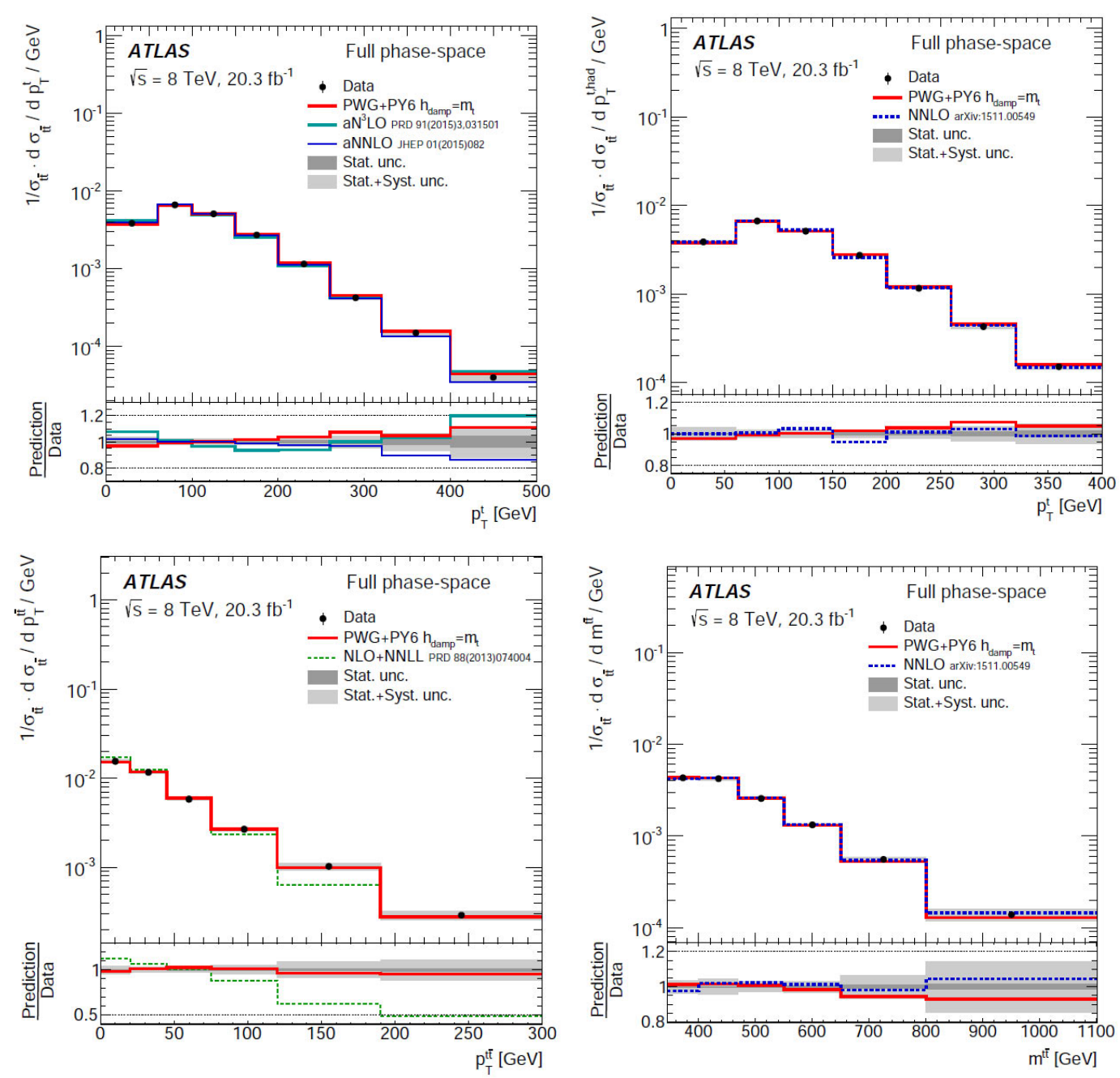

Figure 5. Differential $t \bar{t}$ cross-section measurements at $\sqrt{s}=8 \mathrm{TeV}$ compared with different QCD calculations. Distributions for three different observables are shown: $p_{\mathrm{T}}^{t \text {,had }}, p_{\mathrm{T}}^{t \bar{t}}$ and $m^{t \bar{t}}$ using the full phase space. The bottom panel contains the ratio of prediction to data [15].

unfolding to particle level employs the same Bayesian iterative method as the $8 \mathrm{TeV}$ analysis. Separate migration matrices for the resolved and the boosted topology are used for the unfolding.

The results are in fair agreement with the predictions over a wide kinematic range for the different observables. However, most generators predict harder $p_{\mathrm{T}}$ spectra at high values compared to the observations in data, as it has already been shown by the different Run 1 measurements at $7 \mathrm{TeV}$ and $8 \mathrm{TeV}$. A comparison of the different measurements reveals a similar behaviour of the tested generators at all centre-of-mass energies. Only for Powheg+Herwig++ the agreement with data was better for $8 \mathrm{TeV}$. The total amount of uncertainties is also comparable for different centre-of-mass 
energies. Fig. 6 contains example plots of the measured differential cross-sections for $p_{\mathrm{T}}^{t \text { had }}$ for both the resolved and the boosted topology. The trend of the disagreement between data and prediction for higher transverse momenta is comparable in both topologies. Full NNLO precision leads to a better agreement between data and QCD calculation in all differential measurements presented here.
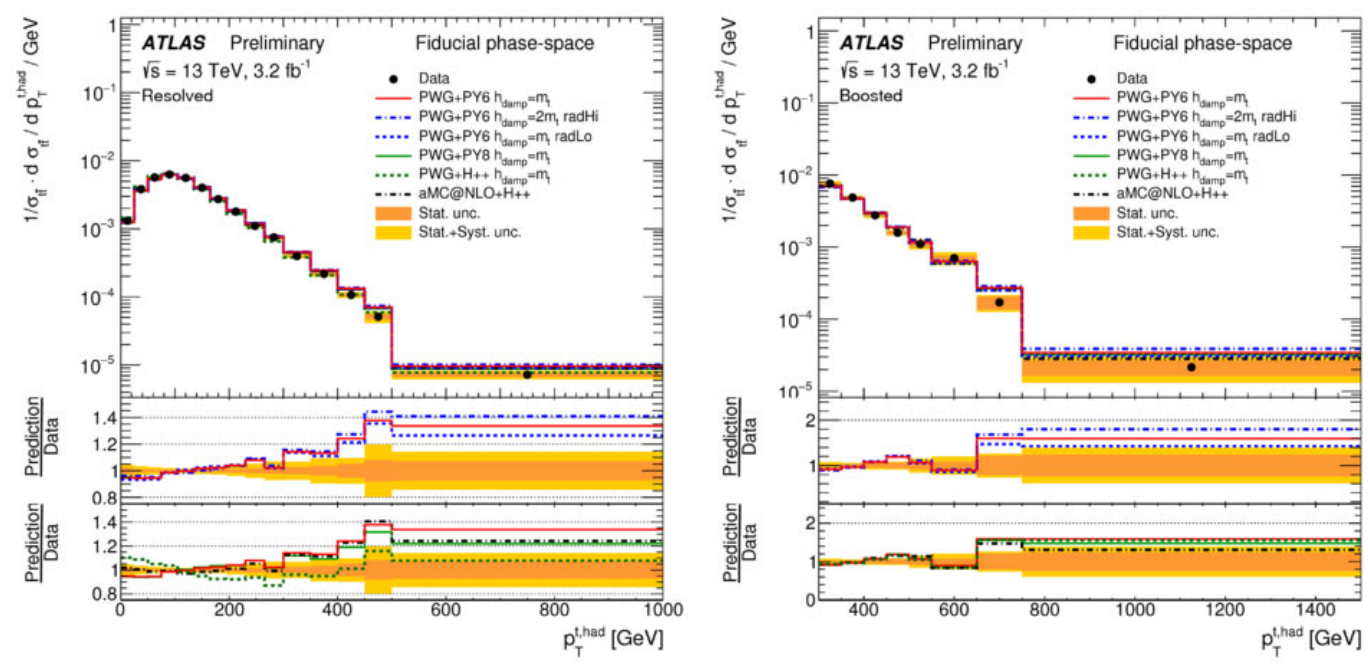

Figure 6. Differential $t \bar{t}$ cross-section measurements at $\sqrt{s}=13 \mathrm{TeV}$ compared with different MC generators. Shown are distributions for the observable $p_{\mathrm{T}}^{t \text {,had }}$ in the resolved and the boosted topology, respectively. The two bottom panels contain the ratios of prediction to data for the different tested predictions [16].

\section{Single Top Wt Production at $8 \mathrm{TeV}$}

A cross-section measurement for the production of a single top quark in association with a $W$ boson at a centre-of-mass energy of $\sqrt{s}=8 \mathrm{TeV}$ [17] with an integrated luminosity of $\mathcal{L}=20.3 \mathrm{fb}^{-1}$ has been performed with data recorded in 2012 with the ATLAS detector. The dilepton channel is used for the analysis according to the following decay chain: $g b \rightarrow W t \rightarrow W^{+} W^{-} b \rightarrow \ell^{+} \ell^{-} v \bar{v} b$. Correspondingly, the selected events are required to contain two charged leptons having opposite sign, missing transverse energy due to the neutrinos and one high- $p_{\mathrm{T}}$ central jet which is $b$-tagged. Boosted decision trees (BDT) are used to optimise the discrimination between signal and background. The cross-section is then extracted using a profile likelihood fit to the BDT response. The dominant uncertainties of this measurement arise from jet-related uncertainties and radiation (initial and final state radiation).

The $W t$ production is observed with $7.7 \sigma$ significance and the cross-section, inclusive of decay modes, is measured to be $\sigma_{W t}=23.0 \pm 1.3$ (stat) ${ }_{-3.5}^{+3.2}$ (syst) \pm 1.1 (lumi) pb, which is consistent with the theoretical QCD calculation based on NLO+NNLL: $22.37 \pm 1.52 \mathrm{pb}$. Furthermore, a lower limit on 0.8 at the $95 \%$ confidence level could be determined for the CKM matrix element $\left|V_{t b}\right|$.

The measurement was also performed in a fiducial phase-space region. The following additional requirements are used: The two leptons need to have a $p_{\mathrm{T}}$ of at least $25 \mathrm{GeV}$ and a pseudo-rapidity of $|\eta|<2.5$. The jet is required to have $p_{\mathrm{T}}>25 \mathrm{GeV}$ and $|\eta|<2.5$. The missing transverse energy needs 
to exceed $20 \mathrm{GeV}$. The final cross-section result amounts to: $\sigma_{W t}^{\text {fid }}=0.85 \pm 0.01$ (stat) ${ }_{-0.07}^{+0.06}$ (syst) \pm 0.03 (lumi) pb. This value is compared to several fiducial cross-section predictions in Fig. 7. The overview displays that the most precise prediction at NLO+NNLL agrees best with the measurement.

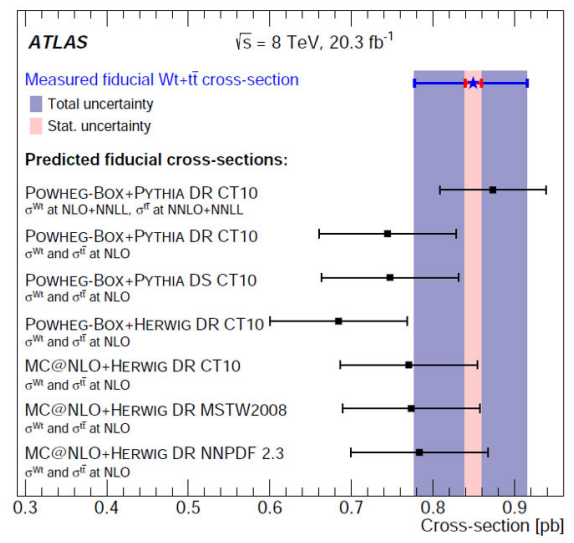

Figure 7. Measured cross-section of $W t$ production in the fiducial region as defined in the text compared to several predicted fiducial cross-sections [17].

\section{Single Top Wt Production at $13 \mathrm{TeV}$}

The measurement of the inclusive cross-section for $W t$ production has been performed at a centreof-mass energy of $\sqrt{s}=13 \mathrm{TeV}$ as well [18]. The dataset taken in 2015 by the ATLAS detector corresponds to an integrated luminosity of $\mathcal{L}=3.2 \mathrm{fb}^{-1}$. As for the corresponding $8 \mathrm{TeV}$ analysis, the dilepton channel is used for this measurement exploiting the decay channel: $g b \rightarrow W t \rightarrow W^{+} W^{-} b \rightarrow$ $\ell^{+} \ell^{-} v \bar{v} b$. Events which are collected thus contain two opposite-sign leptons, one high- $p_{\mathrm{T}}$ central jet which is $b$-tagged and missing transverse energy due to the neutrinos. The analysis technique is similar to the former $8 \mathrm{TeV}$ measurement. Modifications include changes of the BDT training in two regions and the binning of the likelihood fit to extract the cross-section. Furthermore, cuts have been optimised to reject backgrounds more effectively.

The final measured cross-section amounts to $\sigma_{W t}=94 \pm 10$ (stat) ${ }_{-23}^{+28}$ (syst) pb which corresponds to an observed significance of $4.5 \sigma$. This result is consistent with a QCD NLO+NNLL calculation yielding $\sigma_{W t}=71.7 \pm 1.8($ scale $) \pm 3.4(\mathrm{PDF}) \mathrm{pb}$.

\section{Single Top t-Channel Production at $13 \mathrm{TeV}$}

The single top quark production cross-section has also been measured in the $t$-channel using $\mathcal{L}=$ $3.2 \mathrm{fb}^{-1}$ of proton-proton collision data at a centre-of-mass energy of $\sqrt{s}=13 \mathrm{TeV}$, recorded with the ATLAS detector in 2015 [19]. Either top or antitop quarks are produced, illustrated by the Feynman diagrams in Fig. 8. Since the valence $u$ quark density in protons is twice as high as for $d$ quarks, the cross-section for process (a) showing top quark production is expected to be larger than antitop production depicted in (b): $\sigma_{t q}(a)>\sigma_{\bar{t} q}(b)$.

The $\mu+$ jets decay channel is used in this analysis. Hence, events are selected by requiring exactly one muon, missing transverse energy and two high- $p_{\mathrm{T}}$ jets, one of which needs to be $b$-tagged. A 


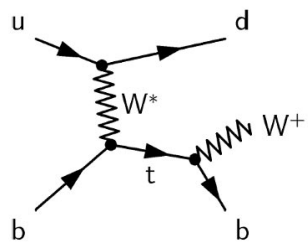

(a)

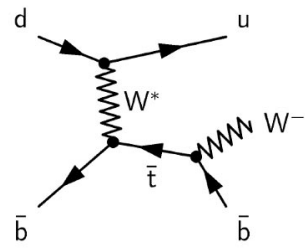

(b)

Figure 8. Feynman diagrams for the two processes of single top $t$-channel production: Either a quark (a) or an antiquark (b) is produced.

binned likelihood fit to a neural network output is employed to extract the desired cross-sections. The dominant sources of systematic uncertainties are due to generator and $b$-tagging uncertainties.

The measured cross-section for top quarks is $\sigma_{t q}=156 \pm 5$ (stat) \pm 27 (syst) \pm 3 (lumi) pb, the one for antitop quarks $\sigma_{\bar{t} q}=91 \pm 4$ (stat) \pm 18 (syst) \pm 2 (lumi) pb. These results are in agreement with an NLO calculation yielding $\sigma_{t q}=136.0_{-4.6}^{+5.4} \mathrm{pb}$ and $\sigma_{\bar{t} q}=81.0_{-3.6}^{+4.1} \mathrm{pb}$, respectively. Besides, a lower limit on the CKM matrix element $\left|V_{t b}\right|$ of 0.84 is obtained at the $95 \%$ confidence level.

\section{Summary}

ATLAS has a robust programme of inclusive and differential cross-section measurements in the top quark sector at 7, 8 and $13 \mathrm{TeV}$. Inclusive cross-sections of top quark pair and single top quark production reach precision levels of up to a few percent uncertainties. Latest $t \bar{t}$ production measurements, also including Tevatron and CMS results, are displayed in Fig. 9 indicating the high precision of recent analyses. The various presented differential cross-section measurements exhibit many interesting features in the understanding of QCD calculations and MC generator predictions. New $13 \mathrm{TeV}$ data recorded in 2016 will allow investigating top quarks with higher energies in even more detail.

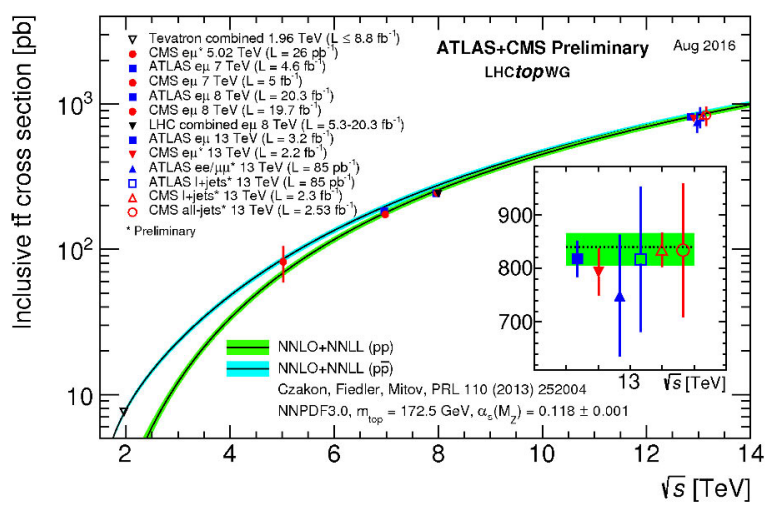

Figure 9. Summary of inclusive $t \bar{t}$ production measurements performed by Tevatron analysis groups, by CMS and by ATLAS at different centre-of-mass energies [20]. 


\section{References}

[1] CDF Collaboration, Phys. Rev. Lett. 74, 2626 (1995)

[2] DØ Collaboration, Phys. Rev. Lett. 74, 2631 (1995)

[3] ATLAS Collaboration, JINST 3, S08003 (2008).

[4] L. Evans and P. Bryant (editors), JINST 3, S08001 (2008).

[5] DØ Collaboration, Phys. Lett. B705, 313 (2011).

[6] DØ Collaboration, Phys. Lett. B726, 656 (2013).

[7] CDF Collaboration, Phys. Rev. Lett. 112, 231804 (2014).

[8] CDF and D0 Collaborations, Phys. Rev. Lett. 112, 231803 (2014).

[9] ATLAS Collaboration, Phys. Lett. B716, 142 (2012).

[10] CMS Collaboration, Phys. Rev. Lett. 110, 022003 (2013).

[11] ATLAS Collaboration, Phys. Lett. B761, 136 (2016).

[12] ATLAS Collaboration, submitted to Phys. Rev. D, arXiv:1607.07281 [hep-ex].

[13] G. D'Agostini, Nucl. Instrum. Meth. A362, 487 (1995).

[14] T. Adye, CERN-2011-006, 313 (2011), arXiv:1105.1160 [physics.data-an].

[15] ATLAS Collaboration, Eur. Phys. J C76, 538 (2016).

[16] ATLAS Collaboration, ATLAS-CONF-2016-040, http://cds.cern.ch/record/2206075.

[17] ATLAS Collaboration, JHEP 01, 064 (2016).

[18] ATLAS Collaboration, ATLAS-CONF-2016-065, http://cds.cern.ch/record/2206207.

[19] ATLAS Collaboration, submitted to JHEP, arXiv:1609.03920 [hep-ex].

[20] https://atlas.web.cern.ch/Atlas/GROUPS/PHYSICS/CombinedSummaryPlots/TOP/ 